OPEN ACCESS

Edited by:

Dorit Ben Shalom,

Ben-Gurion University of the Negev,

Israel

Reviewed by:

Feng Liu,

Tianjin Medical University, China

Dina R. Dajani,

University of Miami, USA

*Correspondence:

$\mathrm{Bin} \mathrm{Hu}$

bh@lzu.edu.cn

Received: 15 June 2016 Accepted: 01 September 2016 Published: 16 September 2016

Citation:

Yao Z, Hu B, Xie Y, Zheng F, Liu G,

Chen $X$ and Zheng W (2016) Resting-State Time-Varying Analysis

Reveals Aberrant Variations of

Functional Connectivity in Autism.

Front. Hum. Neurosci. 10:463.

doi: 10.3389/fnhum.2016.00463

\section{Resting-State Time-Varying Analysis Reveals Aberrant Variations of Functional Connectivity in Autism}

\author{
Zhijun Yao ${ }^{1}$, Bin $\mathrm{Hu}^{1 *}$, Yuanwei Xie ${ }^{1}$, Fang Zheng ${ }^{1}$, Guangyao $\mathrm{Liu}^{2}$, Xuejiao Chen ${ }^{1}$ and \\ Weihao Zheng ${ }^{1}$
}

${ }^{1}$ Ubiquitous Awareness and Intelligent Solutions Lab, School of Information Science and Engineering, Lanzhou University, Lanzhou, China, ${ }^{2}$ Department of Magnetic Resonance, Lanzhou University Second Hospital, Lanzhou, China

Recently, studies based on time-varying functional connectivity have unveiled brain states diversity in some neuropsychiatric disorders, such as schizophrenia and major depressive disorder. However, time-varying functional connectivity analysis of resting-state functional Magnetic Resonance Imaging (fMRI) have been rarely performed on the Autism Spectrum Disorder (ASD). Hence, we performed time-varying connectivity analysis on resting-state fMRI data to investigate brain states mutation in ASD children. ASD showed an imbalance of connectivity state and aberrant ratio of connectivity with different strengths in the whole brain network, and decreased connectivity associated precuneus/posterior cingulate gyrus with medial prefrontal gyrus in default mode network. As compared to typical development children, weak relevance condition (the strength of a large number of connectivities in the state was less than means minus standard deviation of all connection strength) was maintained for a longer time between brain areas of ASD children, and ratios of weak connectivity in brain states varied dramatically in the ASD. In the ASD, the abnormal brain state might be related to repetitive behaviors and stereotypical interests, and macroscopically reflect disruption of gamma-aminobutyric acid the cellular level. The detection of brain states based on time-varying functional connectivity analysis of resting-state fMRI might be conducive for diagnosis and early intervention of ASD before obvious clinical symptoms.

Keywords: fMRI, autism, time-varying, functional connectivity, brain state, divergence

\section{INTRODUCTION}

Autism Spectrum Disorder (ASD) is a lifelong developmental disorder. Children with ASD cannot perform normal social communication, and are characterized by repetitive behaviors and stereotypical interests. Previous studies indicated that impairments in the ASD might reflect deficits in brain network and connectivity (Minshew and Williams, 2007; Vissers et al., 2012; Abbott et al., 2015).

In the neuroimaging analysis, deficits in connectivity have been found to be related to clinical symptoms and behavioral performance in the ASD. Underconnectivity and overconnectivity have been detected in functional connectivity analysis of ASD. In the studies about ASD based on working memory, executive functioning, and response inhibition tasks, the underconnectivity 
of frontal-posterior connections supported the theory that malfunction of circuitry with underconnectivity could cause deficits in integration of information in the brain at cognitive level (Just et al., 2004; Koshino et al., 2005; Just et al., 2007; Kana et al., 2007). In the resting-state studies, overconnectivity was found to be associated with ASD symptom severity (Keown et al., 2013). In the network analysis of ASD, underconnectivity disturbed the integration of network and overconnectivity damaged the segregation of network (Abbott et al., 2015). Integration within brain networks and segregation between them played an important role in the functional brain maturation (Dosenbach et al., 2010). And in the development (from childhood to early adulthood) of brain, negative function connectivities associated with right superior temporal cortex were increased (Kelly et al., 2009).

Recently, dynamic network analysis was introduced in studies of functional connectivity to identify brain connectivity states. Dynamic network analysis could reveal functional connectivity variability during a scan period, which might be impossible in the traditional network analysis of fMRI data. Dynamic network analysis has found some time-varying characteristics of brain connectivity based on fMRI data during a scan period (Liu and Duyn, 2013; Allen et al., 2014; Monti et al., 2014; Yu et al., 2015). Relevant studies have indicated that metastable states identified by dynamic networks corresponded to stages of consciousness (Calhoun et al., 2014). In addition, dynamic network analysis promoted knowledge of actual subnetwork interactions and separation strategies of brain regions (Allen et al., 2014; Yang et al., 2014). In previous studies, dynamic network analysis showed that connectivity state could be shifted in humans with long-term training and experience, such as taxi drivers (Shen et al., 2016). Childhood and adolescence were key stages of brain maturation, and cognitive function networks showed dynamic reorganization in brain maturation (Uddin et al., 2011). And in the development of adolescence, dynamics of brain state was the basis of the development of executive function (Medaglia et al., 2015). ASD might induce deviation of reorganization process from the normal process, and influence connectivity state. In addition, changes of connectivity state in the ASD were less drastic between the resting-state and the tasking-state as compared to typical development (TD) children (Uddin et al., 2015).

Previous functional connectivity fMRI studies showed that overconnectivity and underconnectivity were the major forms of abnormal connectivity in the ASD. To the best of our knowledge, the relationship between connectivity strength and time-varying functional connectivity states in the ASD based on fMRI has not yet been reported. We hypothesized that ASD could influence time-varying functional connectivity states through affecting distribution of connectivity strength and influence the connectivities related to social function. To investigate the influence of ASD on brain connectivity states, we performed group independent component analysis (GICA) and dynamic network analysis on fMRI data of ASD and TD children. GICA can extract spatial distribution of functional regions in the brain.
TABLE 1 | Demographic information of the participants.

\begin{tabular}{lccc}
\hline & TD & ASD & $P$-value \\
\hline$N$ & 44 & 31 & - \\
Age $($ Mean $\pm S D)$ & $12.46 \pm 3.1$ & $11.51 \pm 2.64$ & 0.1693 \\
Gender & Male & Male & - \\
Handedness & Right & Right & - \\
Handedness Score $($ Mean $\pm S D)$ & $62.07 \pm 22.82$ & $63.52 \pm 23.88$ & 0.7914 \\
FIQ Score (Mean $\pm S D)$ & $113.14 \pm 12.32$ & $112.52 \pm 15.87$ & 0.8495 \\
ADI-R Social Total A $($ Mean $\pm S D)$ & - & $18.77 \pm 4.66$ & - \\
ADI-R Verbal Total BV $($ Mean $\pm S D)$ & - & $15.26 \pm 3.84$ & - \\
ADI RRB Total C $($ Mean $\pm S D)$ & - & $5.74 \pm 2.61$ & - \\
ADI R Onset Total D $($ Mean $\pm S D)$ & - & $2.94 \pm 1.34$ & - \\
ADOS Module & - & 3 & - \\
ADOS Total (Mean $\pm S D)$ & - & $11.52 \pm 4.41$ & - \\
ADOS Communication $($ Mean $\pm S D)$ & - & $3.41 \pm 1.74$ & - \\
ADOS Social $($ Mean $\pm S D)$ & - & $8.11 \pm 3.04$ & - \\
ADOS Stereo Behavior $($ Mean $\pm S D)$ & - & $2.67 \pm 1.95$ & - \\
& & &
\end{tabular}

ADI-R, Autism Diagnostic Interview-Revised; ADOS, Autism Diagnostic Observation Schedule; Subjects evaluated by ADOS module 4 were excluded when we calculated mean and SD of ADOS scores.

\section{MATERIALS AND METHODS}

\subsection{Participants and Functional MRI Data Acquisition}

Data of participants were obtained from open accessed dataset collected by NYU Langone Medical Center, a collection site of Autism Brain Image Data Exchange I(ABIDE I) (Di Martino et al., 2014). The site includes 79 (7.1-39.1 years) ASD and 105 TD (6.5-31.8 years) children. The criteria of included subjects are:

(1) male

(2) scores of full intelligence quotient (FIQ, estimated by the fourth subtests of the Wechsler Abbreviated Scale of Intelligence, WASI-IV) above 85

(3) right-handedness

(4) aged 7-18 (not including 18 years old)

TD children were matched with ASD children for age, gender, handedness, FIQ score, and head motion ( $P$-values of the rigid 6 using two-sample $t$-test were $0.7654,0.8762,0.2053,0.6026$, 0.5831 , and 0.6601 , respectively). The detailed demographic information of participants is presented in Table 1. BOLD fMRI data of each participant were acquired with a whole-brain echo planar imaging (EPI) sequence and interleaved slice acquisition $\left(\mathrm{TR}=2 \mathrm{~s}, \mathrm{TE}=15 \mathrm{~ms}\right.$, flip angle $=90^{\circ}$, slice thickness $=$ $4 \mathrm{~mm}, \mathrm{FoV}=240 \mathrm{~mm}, 180$ volumes) on a $3 \mathrm{~T}$ Allegra scanner. Data collections were approved by local IRB of the site, and all data were anonymized. More detailed information is available at http://fcon_1000.projects.nitrc.org/indi/abide/.

\subsection{Data Preprocessing}

Resting-state fMRI raw data were preprocessed by Data Processing Assistant for Resting-State fMRI (DPARSF) (Chao-Gan and Yu-Feng, 2010) based on Statistical Parametric 
Mapping (SPM8). The procedure of preprocessing included removal of first 10 image volumes, realignment, time-slicing and head motion correction, normalization into Montreal Neurological Institute (MNI) standard space, and spatially smoothened by a full-width at half-maximum of $6 \mathrm{~mm}$. All image volumes were aligned to the first volume for each participant in the realignment. In the head motion correction, head motion parameters were estimated according to Friston 24-Parameter Model (Friston et al., 1996). In the normalization, fMRI data were spatially normalized to the MNI EPI template.

\subsection{Independent Component Analysis}

Independent component analysis was performed on the preprocessed fMRI data by GIFT v3.0a using Infomax algorithm (Bell and Sejnowski, 1995), and the order of ICA model was 100. Before performing ICA algorithm, fMRI data dimension reduction was performed by Principal Component Analysis (PCA). The reliability of independent components (ICs) was evaluated by repeating the algorithm 25 times in ICASSO (Himberg and Hyvärinen, 2003). According to spatial distribution of ICs in previous studies (Allen et al., 2014), 54 ICs in seven sub-networks were kept for the following analysis. The seven sub-networks were subcortical (SC), auditory (AU), visual (VIS), somatomotor (SM), cognitive control (CC), default mode (DM), and cerebellar (CB) networks (Figure 1). The other components were related to movement or physical according to their spatial distributions, so they were not included in this study.

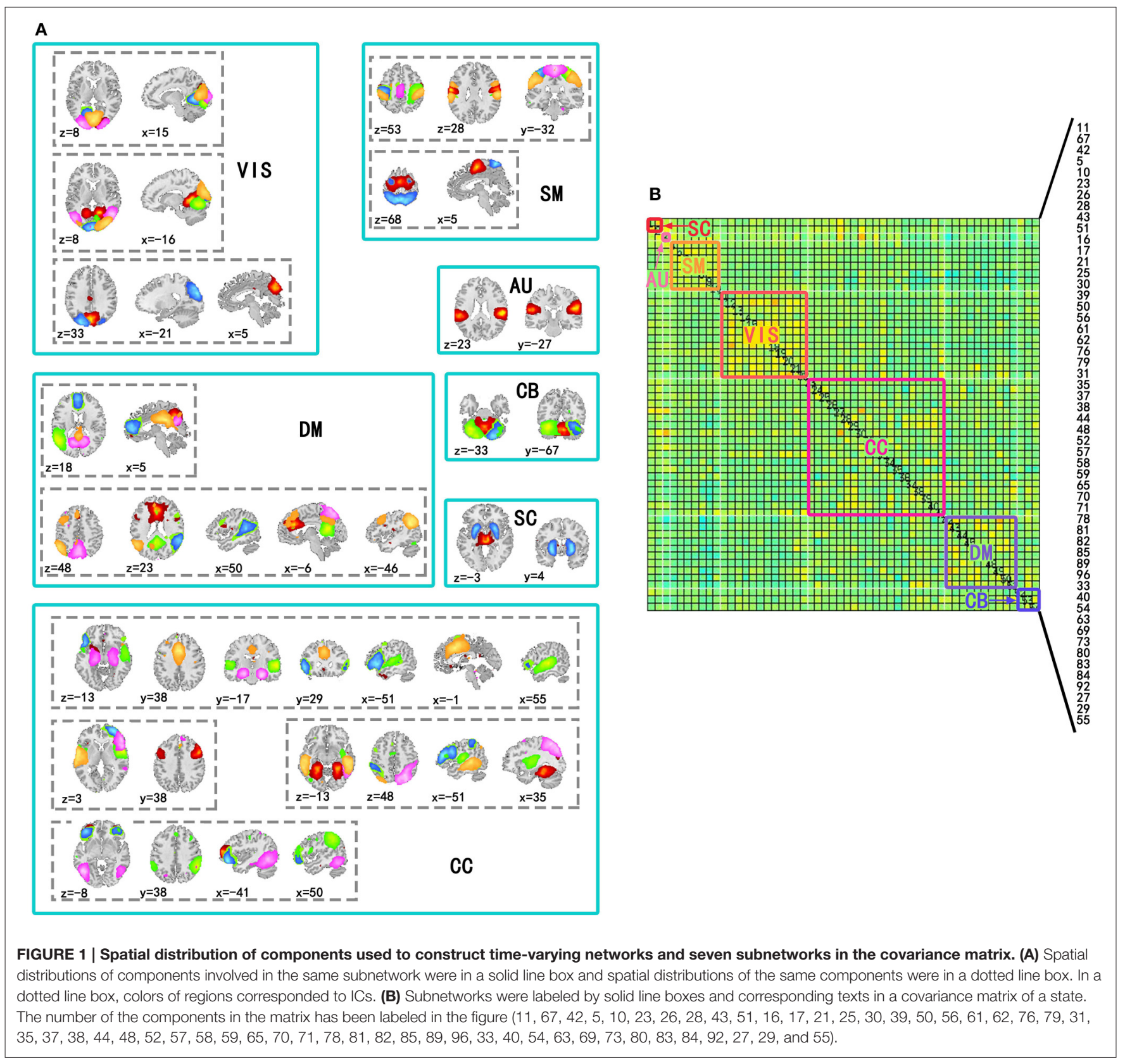




\subsection{Calculation of Time-Varying Connectivity and K-Means Clustering}

The selected ICs were defined as regions of interest (ROIs) to construct the networks. Linear, quadratic, and cubic trends of the time courses extracted according to the ROIs were removed, and six realignment parameters were regressed out. Then, the regressed time courses were depicted by 3 DDESPIKE to remove the outliers, and filtered with a high cutoff frequency of $0.15 \mathrm{~Hz}$ according to the previous study (Allen et al., 2014).

Time-varying functional connectivity was calculated based on segmented time courses in 148 windows created by a tapered window [a rectangle (width $=22$ TRs) with a Gaussian (=3 TRs)] sliding in steps of 1 TR. By calculating Pearson's correlation coefficient (functional connectivity) of all possible ROI pairs in the same sliding window, we constructed covariance matrices $(54 \times 54)$ of each subject. In addition, we used the graphical LASSO (a shrinkage and selection method for linear regression) to evaluate the log-likelihood of covariance matrices, and regularized matrices after evaluating with L1-norm penalty to control sparsity (Friedman et al., 2008).

To determine the connectivity states, covariance matrices of ASD and TD were clustered by k-means clustering algorithm
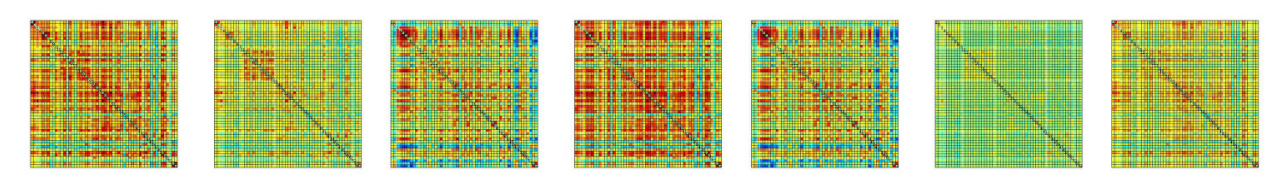

state $=1$
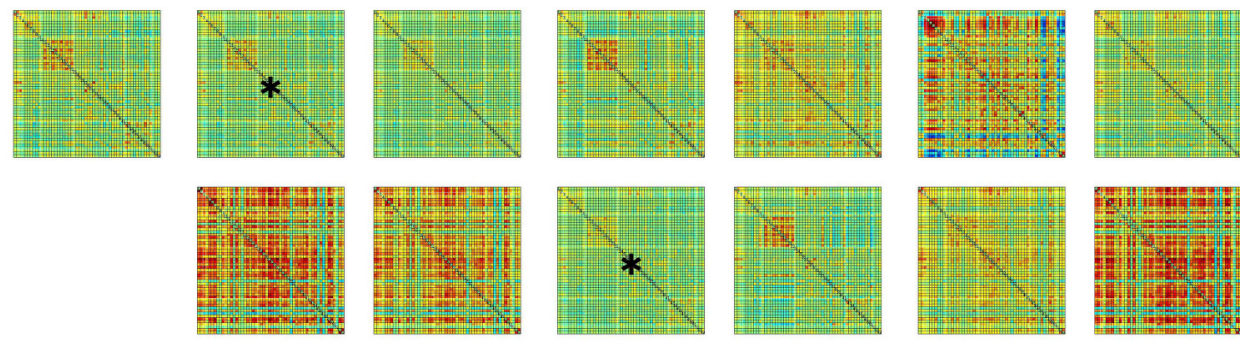

\section{state $=2$}
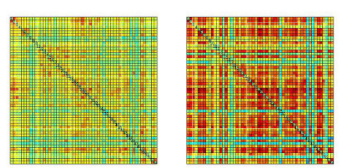

state $=3$
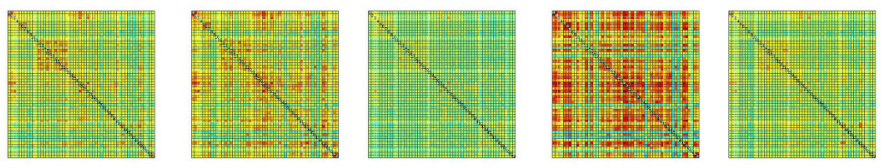

state $=4$
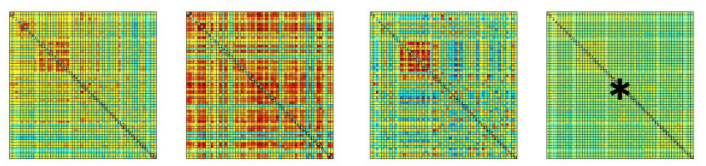

state $=5$
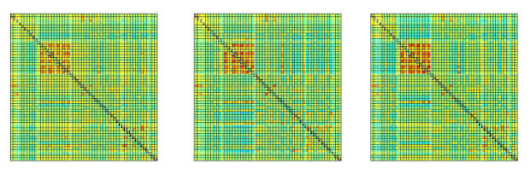

state $=6$
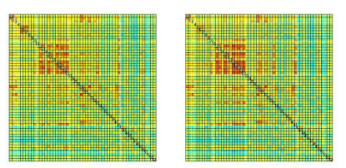

state $=7$

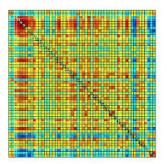

state $=8$

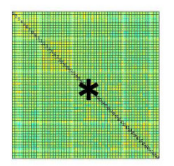

\section{$K=13$}
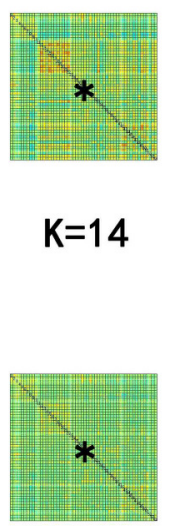

$K=18$
$K=2$
$K=3$
$K=4$
$K=5$
$K=6$
$K=7$
$K=8$

FIGURE 2 | Matrices of all states at $\boldsymbol{k}=\mathbf{2}$ to $\mathbf{8}$ and clustered centroid matrices with significantly different MDTs ( $<<0.05$ ). $K$ was the number of clusters in k-means clustering. This figure showed all centroids of states at $k=2$ to 8 and centroid matrices with significantly different MDTs at $k=13$, 14 , and 18 . MDTs of some states (marked with stars) in the figure were significantly different when values of $\mathrm{k}$ were $3(T=2.1733, p=0.0330<0.05), 5(T=2.1247, p=0.0370<0.05)$, and $8(T=2.2591, p=0.0269<0.05)$. $T$ - and $p$-values were calculated by two-sample $t$-test. In addition, state matrices with significantly different MDTs were marked with star when $\mathrm{k}$ were $13(T=2.6400, p=0.0101<0.05), 14(T=2.3359, p=0.0222<0.05)$, and $18(T=2.0610, p=0.0429<0.05)$. 
TABLE 2 | Percentages for three types of connectivity in all clusters of different $k$-values with significantly different MDTs.

\begin{tabular}{|c|c|c|c|c|c|c|}
\hline \multirow[t]{2}{*}{ Cluster number (k) } & \multicolumn{2}{|c|}{$\begin{array}{c}\text { Percentage of } 1 \\
\text { (weak connectivity) }\end{array}$} & \multicolumn{2}{|c|}{ Percentage of 2} & \multicolumn{2}{|c|}{$\begin{array}{c}\text { Percentage of } 3 \\
\text { (strong connectivity) }\end{array}$} \\
\hline & ASD & NC & ASD & NC & ASD & NC \\
\hline \multirow{3}{*}{3} & 0.5967 & 0.6687 & 0.3275 & 0.2720 & 0.0757 & 0.0591 \\
\hline & 0.7063 & 0.6661 & 0.2598 & 0.2738 & 0.0338 & 0.0600 \\
\hline & 0.5059 & 0.6618 & 0.3073 & 0.2782 & 0.1866 & 0.0599 \\
\hline \multirow{5}{*}{5} & 0.4896 & 0.6593 & 0.2876 & 0.2796 & 0.2226 & 0.0610 \\
\hline & 0.6784 & 0.6512 & 0.2758 & 0.2809 & 0.0457 & 0.0678 \\
\hline & 0.7085 & 0.6704 & 0.2596 & 0.2716 & 0.0318 & 0.0579 \\
\hline & 0.5566 & 0.6695 & 0.3424 & 0.2666 & 0.1008 & 0.0638 \\
\hline & 0.6196 & 0.6691 & 0.3041 & 0.2795 & 0.0761 & 0.0512 \\
\hline \multirow{8}{*}{8} & 0.5285 & 0.6675 & 0.3368 & 0.2740 & 0.1345 & 0.0584 \\
\hline & 0.6573 & 0.6590 & 0.2933 & 0.2764 & 0.0493 & 0.0645 \\
\hline & 0.4531 & 0.6531 & 0.2585 & 0.2817 & 0.2883 & 0.0651 \\
\hline & 0.6296 & 0.6597 & 0.3180 & 0.2722 & 0.0522 & 0.0680 \\
\hline & 0.7198 & 0.6758 & 0.2518 & 0.2698 & 0.0282 & 0.0542 \\
\hline & 0.7018 & 0.6500 & 0.2513 & 0.2801 & 0.0468 & 0.0697 \\
\hline & 0.6216 & 0.6587 & 0.2970 & 0.2864 & 0.0813 & 0.0547 \\
\hline & 0.5290 & 0.7002 & 0.3089 & 0.2605 & 0.1620 & 0.0391 \\
\hline
\end{tabular}

Bold percentages were of weak connectivity in clusters with significantly different MDTs at different k-values.

based on Manhattan distance. Clustered centroid matrices were covariance matrices of connectivity states. We used gap, elbow and Calinski Harabasz to estimate the optimal cluster number. However, optimal cluster number of these methods was two and this was improper. So clustering was performed at $k=2$ to 20 , and repeated 150 times per $k$-value. The effectiveness of the states in the ASD and NC was determined upon the span of states in windows number. In this study, reliable state in the ASD and NC covered at least 10 windows; otherwise, state (covered $<10$ windows) was unreliable. Mean dwell time (MDT) was calculated at each $k$-value. MDT was the average number of windows that were continuous on the time distribution and classified as the same state, representing the duration of each state.

\subsection{Statistical Analysis}

Two-sample $t$-test was used to compare the MDTs of ASD and TD children. To detect the differences of connectivity in each connectivity state, subjects with effective state were included in the two-sample $t$-test for the median covariance matrices of each state. In each $k$-value, median covariance matrices were identified by Manhattan distance priority and tested with the two-sample $t$-test, with a threshold $(p<0.001)$ to identify connectivities with differences. Times of different connectivity at each state for all $k$-values were aggregated. Connectivity with frequency of occurrence $\geq 5$ times was included in the results.

\subsection{Connectivity Strength Discretization}

The absolute values of connectivity strength in all covariance matrices for all $k$-values were divided into three levels (1, 2, and 3) by discretization method based on average and standard deviation of TD $(0.1828 \pm 0.1363)$ and ASD $(0.1698 \pm 0.1322)$ separately. In this study, the connectivity of first level was defined as weak connectivity and last level as strong connectivity. We calculated the percentages for three types of connectivity in all clusters to determine changes in the number of connectivity with different strength in the ASD.

\section{RESULTS}

Significant differences in the MDTs were found when the $k$-values of k-means clustering were $3,5,8,13,14$, and 18. Functional connectivity of ASD children showed weak connectivity for a longer time as compared to TD children according to the $T$ values. Figure 2 shows detailed information of the clustered centroid matrices with significantly different MDTs. Table 2 shows the percentage of three connectivity strength levels at $k$-values with significantly different MDTs. The clusters with maximum ratio of weak connectivity were with significantly different MDTs when $k$ was 3, 5, and 8. However, unreliable clusters existed when $k$-values were $>8$. Figure 3 shows means and standard deviations of percentages for three types of connectivity when $k$-values were from 2 to 8 . Figures 3, 4 indicate less means of weak connectivity in the ASD as compared to TD. Figure 4 also shows that the fluctuation range of percentages for three types of connectivity in the ASD was greater as compared to TD.

The information of abnormal connectivities is presented in Table 3, and the ICs connected by these connectivities are shown in Figure 5, Table 4. The ICs were distributed in cognitive control (ICs: 35, 37, 48, 52, 57, 71, 78, 82), visual (ICs: 25, 30, 50, 56), and default mode (ICs: 40, 83) networks. These ICs mainly involved 

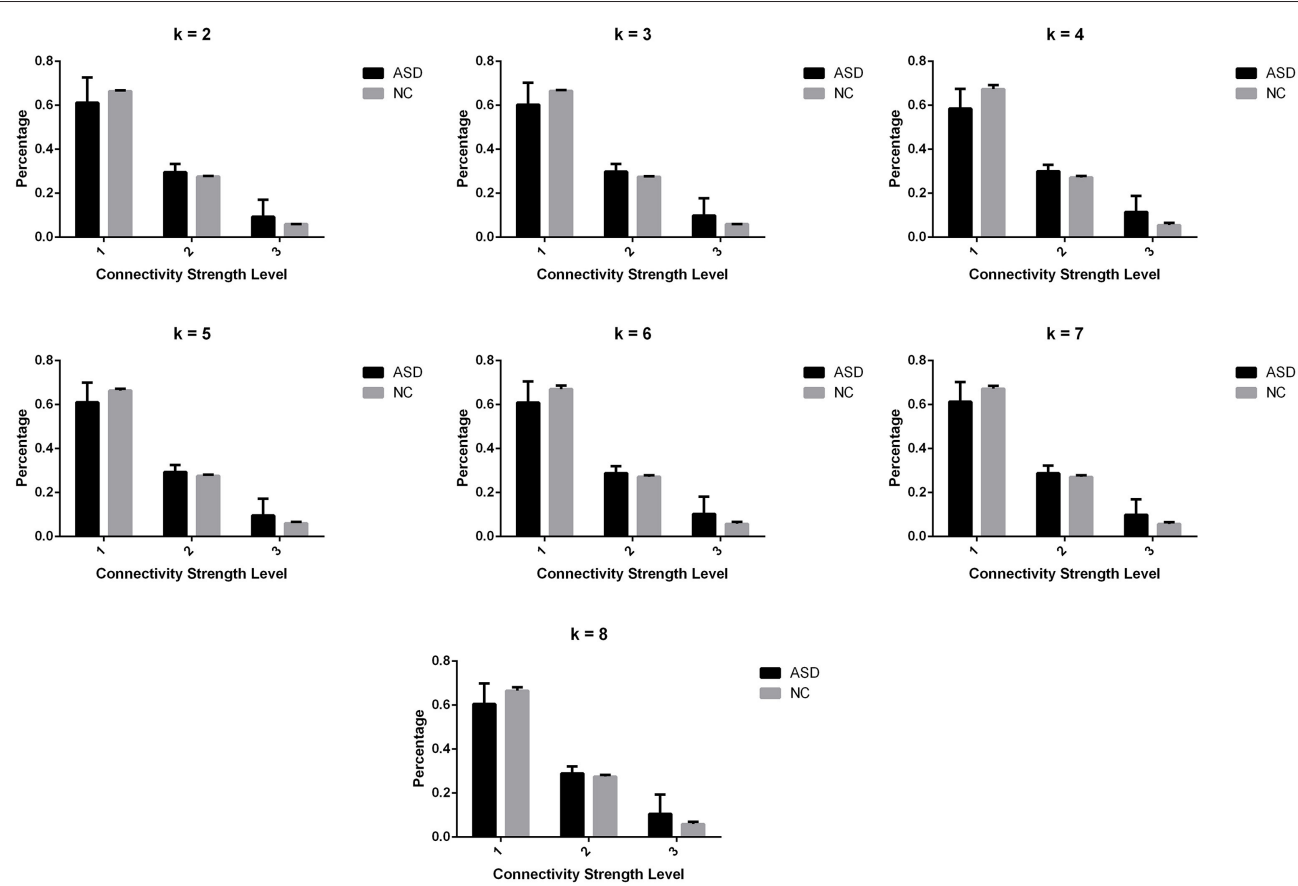

aSD

FIGURE 3 | Means and standard deviations of percentages for three types of connectivity at $k$-values from 2 to 8.

TABLE 3 | The abnormal connectivities with $\geq \mathbf{5}$ times recurrence in the ASD.

\begin{tabular}{lccc}
\hline No. & $\begin{array}{c}\text { Related } \\
\text { components }\end{array}$ & $\begin{array}{c}\text { Frequency of } \\
\text { of occurrence }\end{array}$ & $\begin{array}{c}\text { Increase or } \\
\text { decrease }\end{array}$ \\
\hline 1 & 30,48 & 10 & Increase \\
2 & 37,57 & 10 & Increase \\
3 & 25,71 & 6 & Increase \\
4 & 52,78 & 6 & Decrease \\
5 & 35,50 & 5 & Decrease \\
6 & 40,83 & 5 & Decrease \\
7 & 56,82 & 5 & Decrease \\
\hline
\end{tabular}

MOG.L, CUN.L, frontal lobe (ORBinf.L, IFGoperc.R, MFG.R, and SFGmed.L), right temporal lobe (STG.R and ITG.R), ROL.R, FFG.R, CAL.L, INS.R, and PCUN/PCG in spatial distribution.

\section{DISCUSSION}

The connectivity state in the brain is flexible, which might correspond to diversity of human cognitive functions. Brain connectivity state could change with maturity of the brain, environmental stimulus, and some developmental disorders. And cognitive and behavioral flexibility have been found decreased in the ASD. Also, damages in the connectivities within and between sub-networks (such as default mode, salience, and executive control networks) have been detected in the studies of atypical connectivity patterns and maturation of the ASD (Washington et al., 2014; Abbott et al., 2015).

In the current study, we used time-varying connectivity analysis to detect the impairments of connectivity states in the ASD at resting state. MDTs of brain states with weaker connectivity were abnormal in the ASD (Figure 2). Also, ratios of connectivity with different strengths changed more drastically in the ASD (Figures 3, 4). Drastic changes of connectivity strength might mask task-evoked connectivity changes, and make the brain states undifferentiated (Rubenstein and Merzenich, 2003; Uddin et al., 2015).

In our study, ASD showed more divergent connectivity strength of brain state than TD (Table 2, Figure 2). In the brain, cognitive function depended on connections of specific brain areas. For example, social cognition was related to the prefrontal cortex, the precuneus/posterior cingulate, the hippocampus, the anterior temporal lobes, the posterior superior temporal sulcus and temporo-parietal junction, the fusiform gyrus, the left inferior frontal gyrus, and the anterior insula (Gotts et al., 2012). Hence, some task-evoked functional connectivities were consistent. Based on the consistency, cognitive function state of the brain could be distinguished by whole-brain connectivity patterns (Shirer et al., 2012). Under the same cognitive function, brain states of ASD might diverge from TD due to the disturbed convergence of functional connectivity in the ASD. Also, atypical connectivity patterns of response inhibition were found in previous studies (Kana et al., 2007; Daly et al., 2014).

The abnormal connectivity states may be macroscopical reflection of the excitatory/inhibitory imbalance at the cellular level (Thatcher et al., 2009; Coghlan et al., 2012). In the ASD, 


\section{LEVEL 1}
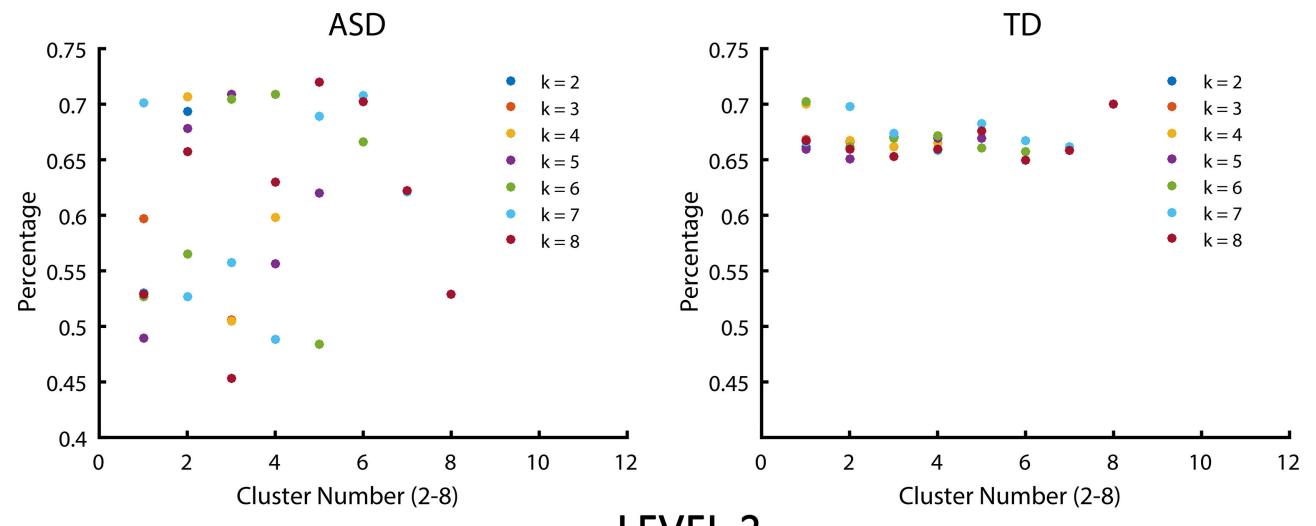

LEVEL 2
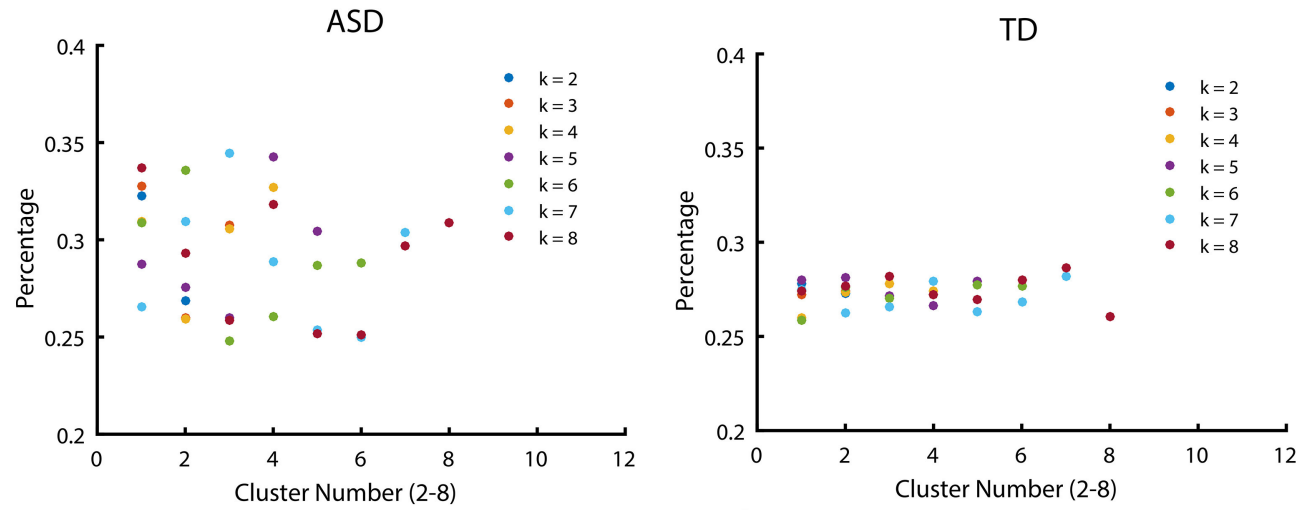

\section{LEVEL 3}
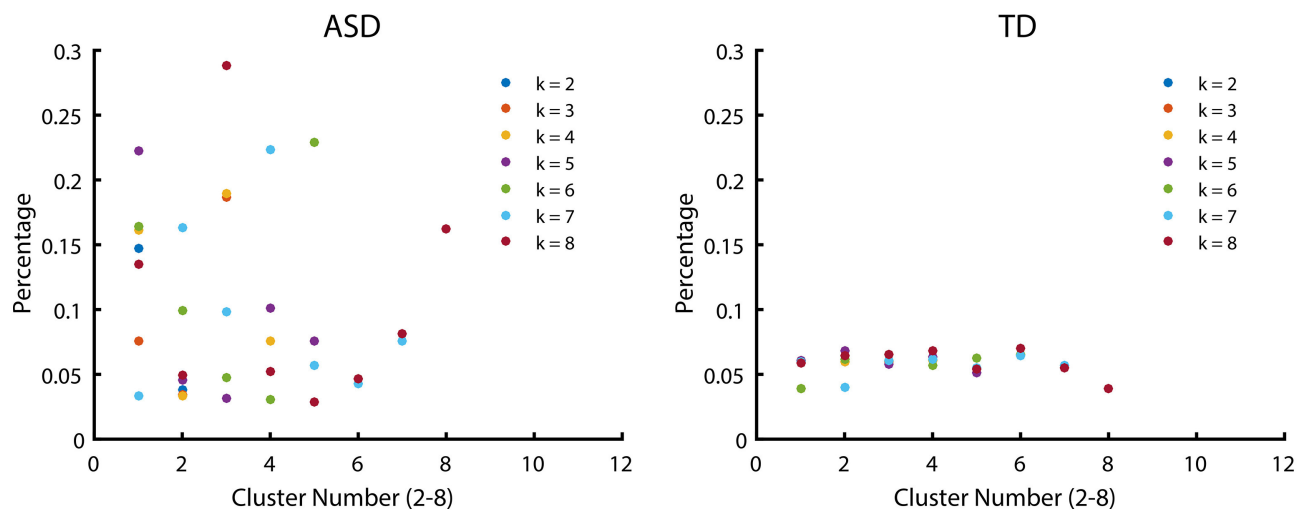

FIGURE $\mathbf{4}$ | Scatter diagrams of percentages for three types of connectivity at $\boldsymbol{k}$-values from $\mathbf{2}$ to $\mathbf{8}$. $K$ was the number of clusters in the clustering process. Level 1, level 2, and level 3 represented three types of connectivity.

stereotypical behavior was found to be related to abnormal gamma-aminobutyric acid (GABA) signaling (Chao et al., 2010). A previous study reported that increased inhibition or decreased excitation at the cellular level might be noise for brain spontaneous activity measured by fMRI, and interfere with neural synchronization of brain in the ASD (Dinstein et al., 2011). The disrupted excitatory/inhibitory balance in the nerve cells might result in disruption of the connectivity on the macro scale, because functional connectivity is a measure of the synchronization between discrete brain regions (Dinstein et al., 2011). In addition, increased connectivity between subcortical and cortical cortices from fMRI studies have been observed as well as decreased ratio of GABA to creatinine in the cerebellum and the primary sensory and motor cortices in the ASD (Gaetz et al., 2014; Rojas et al., 2014; Cerliani et al., 2015). The locations of abnormal connectivity and disrupted 

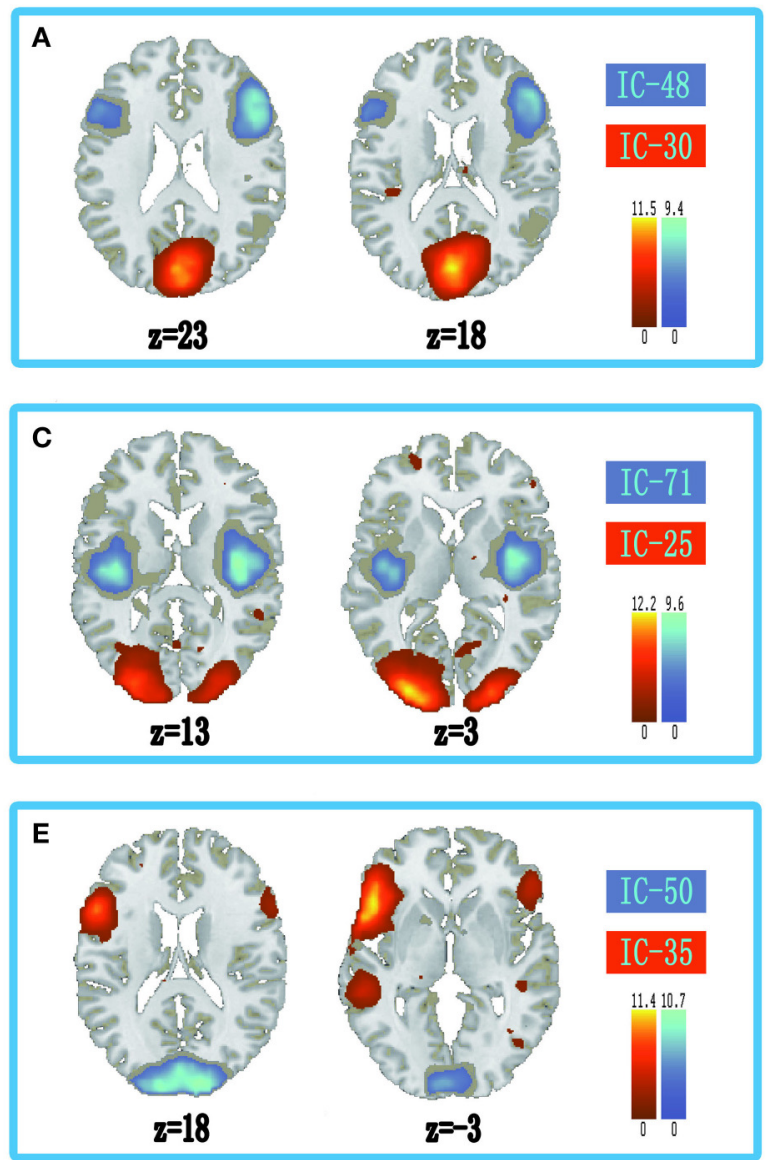
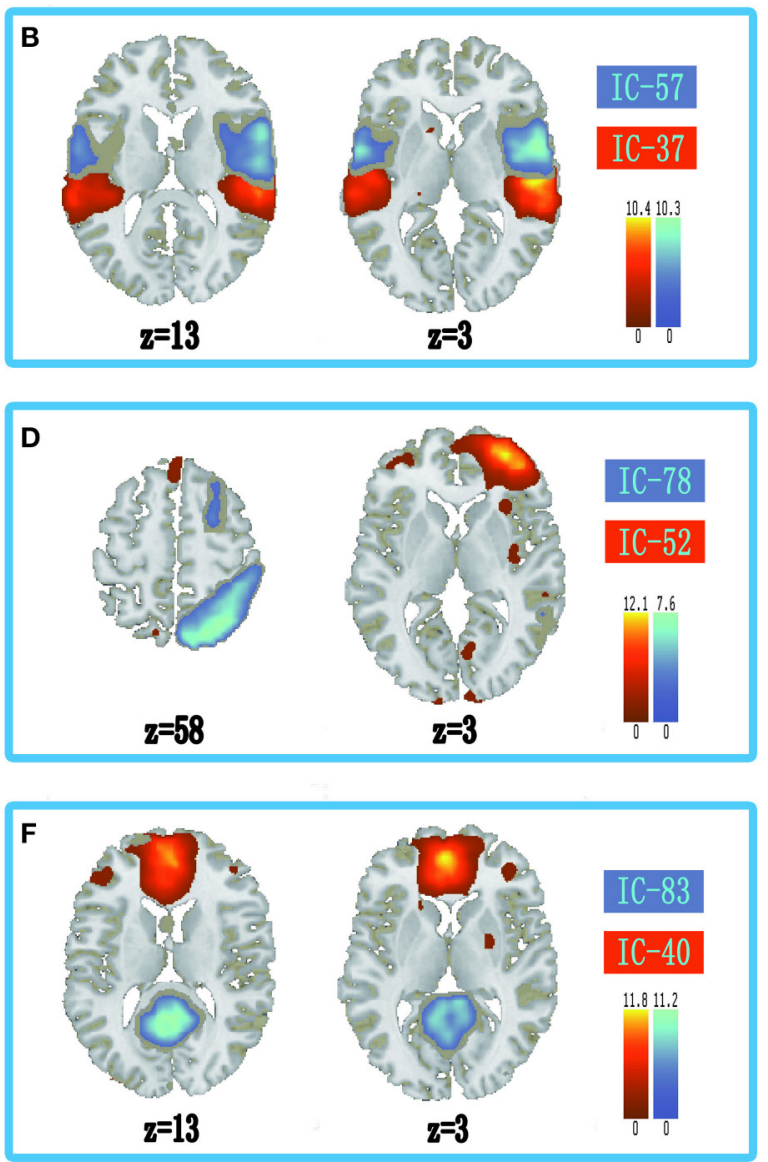

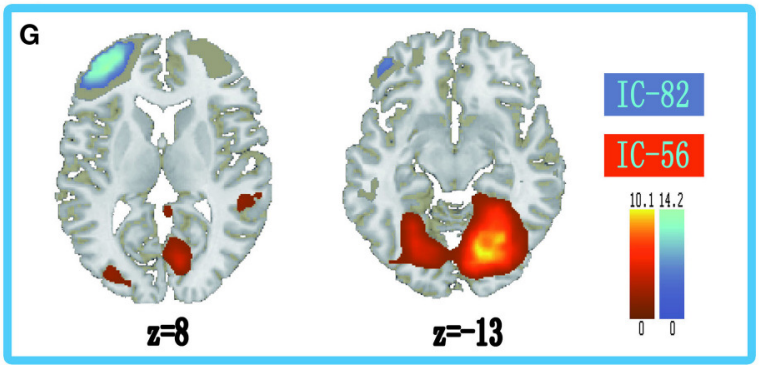

FIGURE 5 | Spatial distribution of independent components linked by abnormal connectivities. The connectivity between independent components in each panel occurred more than five times. (A) Red-left cuneus (IC 30), blue-right inferior frontal gyrus: orbital part (IC 48); (B) Red-right superior temporal gyrus (IC 37), blue-right rolandic operculum (IC 57); (C) Red-left middle occipital gyrus (IC 25), blue-right insula (IC 71); (D) Red-right middle frontal gyrus (IC-52), blue-right inferior temporal gyrus (IC 78); (E) Red-inferior frontal gyrus: orbital part (IC 35), blue-left calcarine sulcus (IC 50); (F) Red-left superior middle frontal gyrus (IC 40),

blue-precuneus/posterior cingulate gyrus (IC 83); (G) Red-right fusiform gyrus (IC 56), blue-left middle frontal gyrus (IC 82).

GABA signaling were consistent, which might indicate that the abnormal brain activities resulted from aberrant GABA signaling in the ASD. Aberrant connectivity status in fMRI might play an important role in the diagnosis of ASD without obvious clinical symptoms.

In the ASD, several cognitive circuits in the brain were aberrant, such as circuits related to visual control, working memory, inhibitory control, emotion processing, face recognition, etc. Some aberrant connectivities in the ASD were connected to social brain, which were the discrete brain regions dominating social cognition (Frith and Frith, 2007; Gotts et al., 2012). In our results, ASD showed decreased connectivity between posterior and frontal regions in DMN (PCUN/PCG and SFGmed.L, Figure 5F). The decreased connectivity was also associated with social deficits, and hampered the ability to maintain a conversation, make eye contact, and perform the pragmatics of language (Assaf et al., 2010; von dem Hagen et al., 2013). In addition, abnormal connectivities in the ASD 
TABLE 4 | Peak values distribution of the independent component spatial maps.

\begin{tabular}{|c|c|c|c|c|c|}
\hline \multirow[t]{2}{*}{ Independent Component } & \multicolumn{3}{|c|}{ Peak MNI coordinate } & \multirow[t]{2}{*}{ Peak intensity (z-score) } & \multirow[t]{2}{*}{ Brain regions } \\
\hline & $x$ & $Y$ & $\boldsymbol{Z}$ & & \\
\hline 25 & -27 & -96 & 3 & 11.3693 & Left middle occipital gyrus (MOG.L) \\
\hline 30 & 0 & -81 & 15 & 10.9039 & Left cuneus (CUN.L) \\
\hline 35 & -48 & 21 & -6 & 11.0173 & Left inferior frontal gyrus, orbital part (ORBinf.L) \\
\hline 37 & 54 & -21 & 3 & 9.7456 & Right superior temporal gyrus (STG.R) \\
\hline 40 & -3 & 54 & 3 & 11.3276 & Left superior frontal gyrus, medial (SFGmed.L) \\
\hline 48 & 54 & 21 & 33 & 8.7204 & Right inferior frontal gyrus, opercular part (IFGoperc.R) \\
\hline 50 & 0 & -99 & 6 & 10.4117 & Left calcarine fissure and surrounding cortex (CAL.L) \\
\hline 52 & 33 & 57 & 3 & 11.2562 & Right middle frontal gyrus (MFG.R) \\
\hline 56 & 18 & -78 & -9 & 9.7335 & Right fusiform gyrus (FFG.R) \\
\hline 57 & 57 & 3 & 3 & 9.5117 & Right rolandic operculum (ROL.R) \\
\hline 71 & 39 & -6 & 6 & 9.1991 & Right insula (INS.R) \\
\hline 78 & 21 & -72 & 54 & 7.2923 & Right inferior temporal gyrus (ITG.R) \\
\hline 82 & -36 & 51 & 9 & 13.4001 & Left middle frontal gyrus (MFG.L) \\
\hline 83 & -3 & -63 & 15 & 10.8304 & Precuneus / Posterior cingulate gyrus (PCUN/PCG) \\
\hline
\end{tabular}

were also found in and between cognitive control (ORBinf.L, IFGoperc.R, INS.R, MFG, STG.R, ITG.R, and ROL.R) and visual networks (left middle occipital gyrus, left cuneus, left calcarine sulcus, and right fusiform gyrus) in our results. The anterior cingulate cortex, ventrolateral prefrontal cortex, dorsolateral prefrontal cortex, and parietal cortex were associated with cognitive control (Solomon et al., 2014). In the human brain, $\mathrm{V} 1$ of visual cortex lies in calcarine sulcus, and motion area of visual cortex is located in the inferior temporal sulcus (Orban et al., 2004). Fusiform gyrus was a key region in face recognition and other social functions (Haxby et al., 2000; Liu et al., 2015). Moreover, CUN was related to control of visual attention and refreshing information in working memory (Makino et al., 2004; Roth and Courtney, 2007; Souliéres et al., 2009). Our results showed decreased connectivity related to visual network in the ASD (Figures 5A,C,E,G). In addition, several studies indicated that frontal lobe and right anterior insula played an important role in inhibitory control (Cai et al., 2014; Daly et al., 2014; Shafritz et al., 2015). Our results showed significantly abnormal connectivities linked to medial prefrontal cortex (MPFC) and superior temporal gyrus in the ASD (Figures 5B,D), which might be related to aberrant activation levels in these brain regions and abnormally implicit emotion processing in the ASD (Kana et al., 2016). These results showed that abnormally activated brain regions induced by tasks might be aberrant at resting-state, which might display targeted behavior modification in the ASD before clinical symptoms.

The disruption of excitation and inhibition balance at connectivity or circuit level might contribute to clinical symptoms in the ASD. Time-varying connectivity analysis in resting-state fMRI can identify the influence of excitation and inhibition balance on whole brain connectivity state, and abnormal connectivity at resting-state in the ASD. However, underlying pathological mechanisms of ASD relied on the study of neurotransmitters in neurons, and relationship between abnormal connectivity and cognitive function might hinder tasking-state neuroimaging and electrophysiological study. In addition, the volume or scan time of samples was relatively small in this study. Prolonging the scan time could capture more accurate metastable states and data dependence of the method affected the universal application of the conclusion. Our study might reflect some characteristics of time-varying functional state in the ASD and the differences of connectivity states in dynamic network analysis between ASD and TD groups might suggest the imbalance between excitation and inhibition.

\section{AUTHOR CONTRIBUTIONS}

$\mathrm{ZY}, \mathrm{BH}$, and $\mathrm{YX}$ conceived and designed the experiments. ZY, YX, and $\mathrm{XC}$ organized and analyzed the raw data. ZY, YX, and GL participated in the statistical analysis and interpretation of data. $\mathrm{ZY}$ and $\mathrm{YX}$ wrote the article, and $\mathrm{BH}, \mathrm{FZ}$, and $\mathrm{WZ}$ revised the manuscript.

\section{FUNDING}

This work was supported by the National Basic Research Program of China (973 Program) (No. 2014CB744600, to BH); the National Natural Science Foundation of China (No. 60973138 and 61003240, to BH); and the International Cooperation Project of Ministry of Science and Technology (No. 2013DFA11140, to $\mathrm{BH})$.

\section{ACKNOWLEDGMENTS}

The authors were very grateful to members of the Autism Brain Imaging Data Exchange for their efforts to aggregated and organized phenotypic data and members of the International Neuroimaging Data-sharing Initiative for their efforts to aggregated and organized imaging data. 


\section{REFERENCES}

Abbott, A. E., Nair, A., Keown, C. L., Datko, M., Jahedi, A., Fishman, I., et al. (2015). Patterns of atypical functional connectivity and behavioral links in autism differ between default, salience, and executive networks. Cereb. Cortex. doi: 10.1093/cercor/bhv191. [Epub ahead of print].

Allen, E. A., Damaraju, E., Plis, S. M., Erhardt, E. B., Eichele, T., and Calhoun, V. D. (2014). Tracking whole-brain connectivity dynamics in the resting state. Cereb. Cortex 24, 663-676. doi: 10.1093/cercor/bhs352

Assaf, M., Jagannathan, K., Calhoun, V. D., Miller, L., Stevens, M. C., Sahl, R., et al. (2010). Abnormal functional connectivity of default mode subnetworks in autism spectrum disorder patients. Neuroimage 53, 247-256. doi: 10.1016/j.neuroimage.2010.05.067

Bell, A. J., and Sejnowski, T. J. (1995). An information-maximization approach to blind separation and blind deconvolution. Neural Comput. 7, 1129-1159. doi: 10.1162/neco.1995.7.6.1129

Cai, W. D., Ryali, S., Chen, T. W., Li, C. S. R., and Menon, V. (2014). Dissociable roles of right inferior frontal cortex and anterior insula in inhibitory control: evidence from intrinsic and task-related functional parcellation, connectivity, and response profile analyses across multiple datasets. J. Neurosci. 34, 14652-14667. doi: 10.1523/JNEUROSCI.304814.2014

Calhoun, V. D., Miller, R., Pearlson, G., and Adali, T. (2014). The chronnectome: time-varying connectivity networks as the next frontier in fmri data discovery. Neuron 84, 262-274. doi: 10.1016/j.neuron.2014.10.015

Cerliani, L., Mennes, M., Thomas, R. M., Di Martino, A., Thioux, M., and Keysers, C. (2015). Increased functional connectivity between subcortical and cortical resting-state networks in autism spectrum disorder. JAMA Psychiatry 72, 767-777. doi: 10.1001/jamapsychiatry.2015.0101

Chao, H. T., Chen, H. M., Samaco, R. C., Xue, M. S., Chahrour, M., Yoo, J., et al. (2010). Dysfunction in gaba signalling mediates autism-like stereotypies and rett syndrome phenotypes. Nature 468, 263-269. doi: 10.1038/nature09582

Chao-Gan, Y., and Yu-Feng, Z. (2010). Dparsf: a matlab toolbox for "pipeline" data analysis of resting-state fmri. Front. Syst. Neurosci. 4:13. doi: 10.3389/fnsys. 2010.00013

Coghlan, S., Horder, J., Inkster, B., Mendez, M. A., Murphy, D. G., and Nutt, D. J. (2012). Gaba system dysfunction in autism and related disorders: from synapse to symptoms. Neurosci. Biobehav. Rev. 36, 2044-2055. doi: 10.1016/j.neubiorev.2012.07.005

Daly, E., Ecker, C., Hallahan, B., Deeley, Q., Craig, M., Murphy, C., et al. (2014). Response inhibition and serotonin in autism: a functional mri study using acute tryptophan depletion. Brain 137, 2600-2610. doi: 10.1093/brain/ awu 178

Di Martino, A., Yan, C. G., Li, Q., Denio, E., Castellanos, F. X., Alaerts, K., et al. (2014). The autism brain imaging data exchange: towards a large-scale evaluation of the intrinsic brain architecture in autism. Mol. Psychiatry 19, 659-667. doi: 10.1038/mp.2013.78

Dinstein, I., Pierce, K., Eyler, L., Solso, S., Malach, R., Behrmann, M., et al. (2011). Disrupted neural synchronization in toddlers with autism. Neuron 70, 1218-1225. doi: 10.1016/j.neuron.2011.04.018

Dosenbach, N. U., Nardos, B., Cohen, A. L., Fair, D. A., Power, J. D., Church, J. A., et al. (2010). Prediction of individual brain maturity using fmri. Science 329, 1358-1361. doi: 10.1126/science.1194144

Friedman, J., Hastie, T., and Tibshirani, R. (2008). Sparse inverse covariance estimation with the graphical lasso. Biostatistics 9, 432-441. doi: 10.1093/biostatistics/kxm045

Friston, K. J., Williams, S., Howard, R., Frackowiak, R. S., and Turner, R. (1996). Movement-related effects in fmri time-series. Magn. Reson. Med. 35, 346-355. doi: $10.1002 / \mathrm{mrm} .1910350312$

Frith, C. D., and Frith, U. (2007). Social cognition in humans. Curr. Biol. 17, R724-R732. doi: 10.1016/j.cub.2007.05.068

Gaetz, W., Bloy, L., Wang, D. J., Port, R. G., Blaskey, L., Levy, S. E., et al. (2014). Gaba estimation in the brains of children on the autism spectrum: measurement precision and regional cortical variation. Neuroimage 86, 1-9. doi: 10.1016/j.neuroimage.2013.05.068

Gotts, S. J., Simmons, W. K., Milbury, L. A., Wallace, G. L., Cox, R. W., and Martin, A. (2012). Fractionation of social brain circuits in autism spectrum disorders. Brain 135(Pt 9), 2711-2725. doi: 10.1093/brain/aws160
Haxby, J. V., Hoffman, E. A., and Gobbini, M. I. (2000). The distributed human neural system for face perception. Trends Cogn. Sci. 4, 223-233. doi: 10.1016/S1364-6613(00)01482-0

Himberg, J., and Hyvärinen, A. (2003). "Icasso: software for investigating the reliability of ica estimates by clustering and visualization," in IEEE 13th Workshop on Neural Networks for Signal Processing, 2003. NNSP'03. 2003 (Toulouse), 259-268.

Just, M. A., Cherkassky, V. L., Keller, T. A., Kana, R. K., and Minshew, N. J. (2007). Functional and anatomical cortical underconnectivity in autism: evidence from an fmri study of an executive function task and corpus callosum morphometry. Cereb. Cortex 17, 951-961. doi: 10.1093/cercor/bhl006

Just, M. A., Cherkassky, V. L., Keller, T. A., and Minshew, N. J. (2004). Cortical activation and synchronization during sentence comprehension in highfunctioning autism: evidence of underconnectivity. Brain 127(Pt 8), 1811-1821. doi: 10.1093/brain/awh199

Kana, R. K., Keller, T. A., Minshew, N. J., and Just, M. A. (2007). Inhibitory control in high-functioning autism: decreased activation and underconnectivity in inhibition networks. Biol. Psychiatry 62, 198-206. doi: 10.1016/j.biopsych.2006.08.004

Kana, R. K., Patriquin, M. A., Black, B. S., Channell, M. M., and Wicker, B. (2016). Altered medial frontal and superior temporal response to implicit processing of emotions in autism. Autism Res. 9, 55-66. doi: 10.1002/aur.1496

Kelly, A. C., Di Martino, A., Uddin, L. Q., Shehzad, Z., Gee, D. G., Reiss, P. T., et al. (2009). Development of anterior cingulate functional connectivity from late childhood to early adulthood. Cereb. Cortex 19, 640-657. doi: 10.1093/cercor/bhn117

Keown, C. L., Shih, P., Nair, A., Peterson, N., Mulvey, M. E., and Muller, R. A. (2013). Local functional overconnectivity in posterior brain regions is associated with symptom severity in autism spectrum disorders. Cell Rep. 5, 567-572. doi: 10.1016/j.celrep.2013.10.003

Koshino, H., Carpenter, P. A., Minshew, N. J., Cherkassky, V. L., Keller, T. A., and Just, M. A. (2005). Functional connectivity in an fmri working memory task in high-functioning autism. Neuroimage 24, 810-821. doi: 10.1016/j.neuroimage.2004.09.028

Liu, F., Zhu, C., Wang, Y., Guo, W., Li, M., Wang, W., et al. (2015). Disrupted cortical hubs in functional brain networks in social anxiety disorder. Clin. Neurophysiol. 126, 1711-1716. doi: 10.1016/j.clinph.2014.11.014

Liu, X., and Duyn, J. H. (2013). Time-varying functional network information extracted from brief instances of spontaneous brain activity. Proc. Natl. Acad. Sci. U.S.A. 110, 4392-4397. doi: 10.1073/pnas.1216856110

Makino, Y., Yokosawa, K., Takeda, Y., and Kumada, T. (2004). Visual search and memory search engage extensive overlapping cerebral cortices: an fmri study. Neuroimage 23, 525-533. doi: 10.1016/j.neuroimage.2004.06.026

Medaglia, J. D., Satterthwaite, T. D., Moore, T. M., Ruparel, K., Gur, R. C., Gur, R. E., et al. (2015). Flexible traversal through diverse brain states underlies executive function in normative neurodevelopment. arXiv:1510.08780.

Minshew, N. D., and Williams, D. L. (2007). The new neurobiology of autism: cortex, connectivity, and neuronal organization. Arch. Neurol. 64, 945-950. doi: 10.1001/archneur.64.10.1464

Monti, R. P., Hellyer, P., Sharp, D., Leech, R., Anagnostopoulos, C., and Montana, G. (2014). Estimating time-varying brain connectivity networks from functional mri time series. Neuroimage 103, 427-443. doi: 10.1016/j.neuroimage.2014.07.033

Orban, G. A., Van Essen, D., and Vanduffel, W. (2004). Comparative mapping of higher visual areas in monkeys and humans. Trends Cogn. Sci. 8, 315-324. doi: 10.1016/j.tics.2004.05.009

Rojas, D. C., Singel, D., Steinmetz, S., Hepburn, S., and Brown, M. S. (2014). Decreased left perisylvian gaba concentration in children with autism and unaffected siblings. Neuroimage 86, 28-34. doi: 10.1016/j.neuroimage.2013.01.045

Roth, J. K., and Courtney, S. M. (2007). Neural system for updating object working memory from different sources: Sensory stimuli or long-term memory. Neuroimage 38, 617-630. doi: 10.1016/j.neuroimage.2007.06.037

Rubenstein, J. L. R., and Merzenich, M. M. (2003). Model of autism: increased ratio of excitation/inhibition in key neural systems. Genes Brain Behav. 2, 255-267. doi: 10.1034/j.1601-183X.2003.00037.x

Shafritz, K. M., Bregman, J. D., Ikuta, T., and Szeszko, P. R. (2015). Neural systems mediating decision-making and response inhibition for social and nonsocial 
stimuli in autism. Progr. Neuro-Psychopharmacol. Biol. Psychiatry 60, 112-120. doi: 10.1016/j.pnpbp.2015.03.001

Shen, H., Li, Z., Qin, J., Liu, Q., Wang, L., Zeng, L.-L., et al. (2016). Changes in functional connectivity dynamics associated with vigilance network in taxi drivers. Neuroimage 124, 367-378. doi: 10.1016/j.neuroimage.2015.09.010

Shirer, W. R., Ryali, S., Rykhlevskaia, E., Menon, V., and Greicius, M. D. (2012). Decoding subject-driven cognitive states with whole-brain connectivity patterns. Cereb. Cortex 22, 158-165. doi: 10.1093/cercor/bhr099

Solomon, M., Yoon, J. H., Ragland, J. D., Niendam, T. A., Lesh, T. A., Fairbrother, W., et al. (2014). The development of the neural substrates of cognitive control in adolescents with autism spectrum disorders. Biol. Psychiatry 76, 412-421. doi: 10.1016/j.biopsych.2013.08.036

Souliéres, I., Dawson, M., Samson, F., Barbeau, E. B., Sahyoun, C. P., Strangman, G. E., et al. (2009). Enhanced visual processing contributes to matrix reasoning in autism. Hum. Brain Mapp. 30, 4082-4107. doi: 10.1002/hbm.20831

Thatcher, R. W., North, D. M., Neubrander, J., Biver, C. J., Cutler, S., and DeFina, P. (2009). Autism and eeg phase reset: deficient gaba mediated inhibition in thalamo-cortical circuits. Dev. Neuropsychol. 34, 780-800. doi: 10.1080/87565640903265178

Uddin, L. Q., Supekar, K., Lynch, C. J., Cheng, K. M., Odriozola, P., Barth, M. E., et al. (2015). Brain state differentiation and behavioral inflexibility in autism. Cereb. Cortex 25, 4740-4747. doi: 10.1093/cercor/bhu161

Uddin, L. Q., Supekar, K. S., Ryali, S., and Menon, V. (2011). Dynamic reconfiguration of structural and functional connectivity across core neurocognitive brain networks with development. J. Neurosci. 31, 18578-18589. doi: 10.1523/JNEUROSCI.4465-11.2011

Vissers, M. E., Cohen, M. X., and Geurts, H. M. (2012). Brain connectivity and high functioning autism: a promising path of research that needs refined models, methodological convergence, and stronger behavioral links. Neurosci. Biobehav. Rev. 36, 604-625. doi: 10.1016/j.neubiorev.2011.09.003

von dem Hagen, E. A., Stoyanova, R. S., Baron-Cohen, S., and Calder, A. J. (2013). Reduced functional connectivity within and between social resting state networks in autism spectrum conditions. Soc. Cogn. Affect. Neurosci. 8, 694-701. doi: 10.1093/scan/nss053

Washington, S. D., Gordon, E. M., Brar, J., Warburton, S., Sawyer, A. T., Wolfe, A., et al. (2014). Dysmaturation of the default mode network in autism. Hum. Brain Mapp. 35, 1284-1296. doi: 10.1002/hbm.22252

Yang, Z., Craddock, R. C., Margulies, D. S., Yan, C. G., and Milham, M. P. (2014). Common intrinsic connectivity states among posteromedial cortex subdivisions: insights from analysis of temporal dynamics. Neuroimage 93, 124-137. doi: 10.1016/j.neuroimage.2014.02.014

Yu, Q. B., Erhardt, E. B., Sui, J., Du, Y. H., He, H., Hjelm, D., et al. (2015). Assessing dynamic brain graphs of time-varying connectivity in fmri data: application to healthy controls and patients with schizophrenia. Neuroimage 107, 345-355. doi: 10.1016/j.neuroimage.2014.12.020

Conflict of Interest Statement: The authors declare that the research was conducted in the absence of any commercial or financial relationships that could be construed as a potential conflict of interest.

Copyright (C) 2016 Yao, Hu, Xie, Zheng, Liu, Chen and Zheng. This is an open-access article distributed under the terms of the Creative Commons Attribution License (CC $B Y)$. The use, distribution or reproduction in other forums is permitted, provided the original author(s) or licensor are credited and that the original publication in this journal is cited, in accordance with accepted academic practice. No use, distribution or reproduction is permitted which does not comply with these terms. 